Gelder, M., GATH, D. \& Mayou, R. (1989) Oxford Textbook of Psychiatry (2nd edn). Oxford: Oxford University Press.

Albert Michael

P. Ninan KuRIaN

St Finian's Hospital

Portlaoise

Co. Laois, Eire

\section{Is schizophrenia disappearing?}

SIR: Could the fall in rate of diagnosis of schizophrenia be linked with the emergence of child and adolescent psychiatry?

During the time under consideration by Eagles (Journal, June 1991, 158, 834-835), there has been a proliferation of what might collectively be called mental health services for children and adolescents. A few were in existence before World War II but following the 1944 Education Act, and generally with the increasing acceptability of such services, there was a vast expansion of child and adolescent psychiatric services, child guidance services, school psychological services, special schools for disturbed children and better psychological care in local authority children's homes. Would these not have had some impact on reducing the incidence of adult mental illness, including schizophrenia?

There were similar increases in such services throughout Europe at much the same time; the USA was always somewhat ahead of Europe.

The emergence of child and adolescent psychiatry has been considered sufficiently important for all candidates sitting the membership exam of the Royal College of Psychiatrists to have to demonstrate some knowledge of child and adolescent psychiatry. It is my experience that this greater awareness of the emotional disorders of young people shown by the majority of psychiatrists has led increasingly to their referral to appropriate services rather than to admission to adult mental hospitals; a regrettable practice that occurred all too often in the past.

\section{Windermere Road \\ Muswell Hill, London N10 2RD}

Robin BenIaNS

Lateral ventricular size, educational level and patient subtypes in schizophrenia

SIR: Since the first report of lateral ventricular enlargement in schizophrenia, a number of studies have addressed this issue, reporting positive findings in general (for a review see Shelton \& Weinberger, 1986).

Differences in the population from which schizophrenics are drawn are widely recognised as possibly affecting neuromorphological findings (Luchins,
1982) and severe and chronic forms of schizophrenia are reported to show greater computerised tomography (CT) abnormalities (Goldberg et al, 1988). Educational status also seems to affect neuromorphological measurements obtained through brain imaging techniques (for a discussion see Andreasen, 1988).

In order to see how the choice of patient subtypes and educational level matching could be related to the finding of statistically significant differences in neuromorphological measurements between schizophrenics and healthy subjects, we have compared the mean ventricular brain ratio (VBR) in a controlled magnetic resonance imaging (MRI) study (Rossi et al, 1990a) and a subsequent MRI replication study (Rossi et al, 1990b).

In the first study, patients and controls were matched for age (within three years) and sex, but not for educational level, whereas patients in the second study were also strictly matched educationally (within one year) with the controls. The first patient group consisted of 39 patients, 33 of whom were relapsing patients with a relatively good outcome, all able to live in the community with out-patient care, and six severe patients who met the operational criteria of Keefe et al (1987) for Kraepelin's dementia praecox. The mean VBR for the overall patient group was 5.1; for the Kraepelinian subgroup it was 6.8 and for the relapsing subgroup it was 4.8 . The mean age of the 39 patients was 31.23 years (s.d. 7.04) and their mean length of illness was 6.97 years (s.d. 4.69); mean educational levels of patients and controls were 10.51 (s.d. 3.03) and 17.03 (s.d. 2.42) years respectively. The Kraepelinian and relapsing patients had a mean educational level of 10.33 (s.d. 3.32) and 10.54 (s.d. 3.03) years, respectively.

The second patient group was represented only by relapsing patients, living in the community, with a mean age of 26.47 years (s.d. 4.82) and a length of illness of 4.41 years (s.d. 2.50). Their mean educational level was 10.94 (s.d. 4.19) years and that of the matched healthy controls 11.61 (s.d. 3.15) years.

The mean values in the two studies are slightly different but they were conducted with different MRI machines and by different raters, so between-study comparisons cannot be made. The first MRI study shows a statistically significant VBR increase in the overall patient group when compared with controls (two-tailed $t$-test, $t=2.56, P<0.01$ ). When the Kraepelinian patients were excluded, this difference was no more significant, as was the case in the replication MRI study. Nonetheless, the residual VBR difference showed a strong trend towards significance (two-tailed $t$-test, $t=1.89, P<0.06$ ), which might be due to differences in educational level 